\title{
Pharyngeal Obturator Prosthetic Rehabilitation of Velopharyngeal Insufficiency
}

\author{
Muhammad Waseem Ullah Khan, Muhammad Asif Mushtaq and Asif Ali Shah \\ Department of Prosthodontics, de'Montmorency College of Dentistry / Punjab Dental Hospital, Lahore, Pakistan
}

\begin{abstract}
Congenital or acquired defects of soft palate cause physical, functional and psychological disabilities. Surgical closure or prosthetic rehabilitation are the two treatment modalities. If surgery is contraindicated, prosthetic rehabilitation is the alternative treatment option. Pharyngeal obturator prosthesis provides adequate closure of the velopharyngeal insufficiency. In this case report, a young patient is treated for velopharyngeal insufficiency with pharyngeal obturator as the patient had refused to undergo surgical closure. The prosthesis was successfully fabricated and was evaluated for proper functioning. It improved speech, deglutition and psychological well-being of the patient.
\end{abstract}

Key Words: Pharyngeal obturator, Velopharyngeal insufficiency, Hypernasality, Maxillofacial prosthodontics.

How to cite this article: Khan MWU, Mushtaq MA, Shah AA. Pharyngeal obturator prosthetic rehabilitation of velopharyngeal insufficiency. J Coll Physicians Surg Pak 2019; 29(12):1225-1227.

\section{INTRODUCTION}

The velopharyngeal sphincter is a three-dimensional complex muscular structure formed anteriorly by soft palate, posteriorly by posterior pharyngeal wall and laterally by lateral pharyngeal wall.1,2 This intricate muscular valve acts as a dynamic separator between nasal and oral cavity, which is mandatory for pertinent phonation and swallowing. Compromised function of this valve leads to grievous consequences. ${ }^{3}$ Soft palate abnormalities are divided into three categories. Congenital abnormalities include cleft palate, acquired abnormalities include trauma and surgical resection of the soft palate due to any pathology, and developmental abnormalities, either muscular or neurological, causing diminished capacity of soft palate to respond to functional demands. ${ }^{4}$ The term velopharyngeal dysfunction (VPD) describes the whole spectrum of problems with the velopharyngeal sphincter mechanism. Velopharyngeal insufficiency refers to structural abnormality leading to inadequate velopharyngeal closure. Velopharyngeal incompetence refers to the dysfunction of velopharyngeal sphincter because of neurologic impairment, but velum is structurally complete. Velopharyngeal mislearning refers to the VPD that is exclusively functional without physiologic or anatomic etiologic factors for errors in articulation. $^{2}$

Correspondence to: Dr. Muhammad Asif Mushtaq, Department of Prosthodontics, de'Montmorency College of Dentistry / Punjab Dental Hospital, Forl Road, Lahore, Pakistan

E-mail:drasif100@yahoo.com

Received: January 05, 2019; Revised: March 10, 2019; Accepted: March 28, 2019
In velopharyngeal insufficiency, leakage of airflow occurs to nasal cavity and causes deficiency in the formation of nasal sounds. Thus, it causes hypernasality, decreased intraoral pressure for pressure consonants and audible nasal emission leading to impaired speech articulation and difficulty in communication due to distorted speech. Nasal regurgitation of food particles during deglutition leads to difficulty in swallowing. These problems cause disturbed social life of patients leading to psychological disturbances. 3,5 To improve the physical and social wellbeing of patients, surgery along with speech therapy is the common treatment approach. But there are various conditions when surgery is not possible, e.g. advanced cardiovascular or neurologic disease, biomechanical limitations (microstomia or limited mouth opening), anomalous medical deviation of internal carotid artery, presence or risk of obstructive sleep apnea or patient refusing surgery because of economic or psychological reasons. In such circumstances, treatment of choice will be prosthetic rehabilitation along with speech therapy.3,6

Prosthetic management of velopharyngeal insufficiency involves placement of pharyngeal obturator and palatal lift prosthesis for velopharyngeal incompetence. Pharyngeal obturator is a removable maxillary prosthesis which has a posterior extension to separate oropharynx and nasopharynx. It provides a fixed structure against which the pharyngeal muscles can function. This prosthesis rehabilitate the soft palate structure and adequate velopharyngeal closure is obtained leading to improved speech, deglutition and appearance. 3,7

We are reporting a case of congenital velopharyngeal insufficiency. The patient refused to undergo surgical treatment, so his defect was restored with pharyngeal obturator. 


\section{CASE REPORT}

A 25-year male presented to the Department of Prosthodontics with the complaint of difficulty in speech and verbal communication. The speech of the patient was hypernasal since the time of childhood. Patient was a known case of cleft of the soft palate. He had undergone surgical repair of the soft palate in his childhood. Otherwise, he was medically fit. On extraoral examination, there were no abnormal facial features, surgical scars, deformities or facial asymmetry.

On intraoral examination, velopharyngeal insufficiency was revealed because of the cleft of soft palate. The cleft was throughout the soft palate along with bifurcated uvula (Figure 1). The dentition of the patient was healthy. $\mathrm{He}$ had never received speech therapy before. He was trying to talk in low tone to decrease nasal emission of air. On clinical assessment of speech difficulty, hypernasality and increased nasal emission was detected while performing speech, swallowing and respiration. The speech pathologist had assessed inappropriate nasal air emission, resonance and articulation. Patient had never used any obturator before because of lack of knowledge about available treatment options.

Patient had refused to undergo any surgical procedure for the correction of this velopharyngeal insufficiency. A pharyngeal obturator prosthesis, also known as speech bulb prosthesis was recommended with clasp retention. Initial assessment of dentition and periodontium was performed. Preliminary diagnostic impression was made with irreversible hydrocolloid (Cavex CA 37, Cavex Holland BV, Netherlands). Bulb shaped design of the palatal part of the prosthesis was drawn on diagnostic cast. Mouth preparations were carried out for retentive part of the prosthesis. After mouth preparation, modified stock tray was used to record the final impression of the palate and preliminary for the soft palate with irreversible hydrocolloid. The master cast was fabricated. Palatal part of the prosthesis was designed and casted in metal alongwith a small meshwork for the functional impression of the pharyngeal part of the prosthesis (Figure 1).

Low fusing impression compound (Harvard impression compound, Harvard Dental International $\mathrm{GmbH}$, Germany) was added to the retentive meshwork to record the details of the defect. Later on, incremental addition or removal of the low fusing impression material was done during border molding. Border molding was achieved by asking the patient to move his head in rotatory manner from side to side, flexion and extension of neck and to say "ah" and swallow his saliva.

After performing border molding with impression compound, $1-1.5 \mathrm{~mm}$ of the surface of the impression compound was scraped off. Tissue conditioner was applied to the surface of pharyngeal bulb and all the functional movements were repeated. The material was left in mouth for 8-10 minutes (Figure 2). Later, acrylic bulb was fabricated from that impression of the pharyngeal part of the prosthesis (Figure 3). During laboratory processing, careful attention was given to provide good retention of acrylic to palatal metal meshwork.

At the time of delivery of the prosthesis to patient, pressure indicating paste was applied to the pharyngeal surface of the obturator to assess any undue pressure areas and difficulty in respiration. After delivering the prosthesis to patient, post-insertion instructions were given for the care of the prosthesis.

After the completion of the prosthetic rehabilitation, patient was referred to speech pathologist. According to the assessment of speech pathologist, the plosive consonants such as $b, p$ and normal consonants such as $\mathrm{m}, \mathrm{n}$ and ng were properly articulated. There was no hypernasality or hyponasality. During one year of followup period, patient's well-being improved markedly, both socially and psychologically, as assessed by speech therapist.

\section{DISCUSSION}

Location, size and nature of velopharyngeal insufficiency determines the nature of treatment strategies. Perceptual speech assessment is the most reliable method of diagnosis of velopharyngeal insufficiency because it can not only determine the existence of problem but can also determine the size of velopharyngeal gap. ${ }^{3}$ Surgical correction of velopharyngeal insufficiency along with

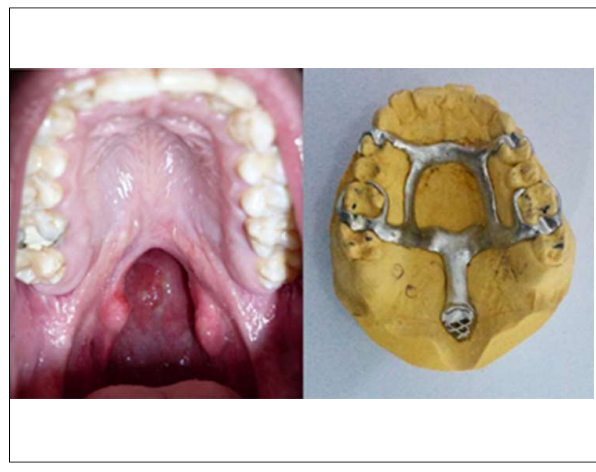

Figure 1: Preoperative clinical picture and master cast with metal framework.

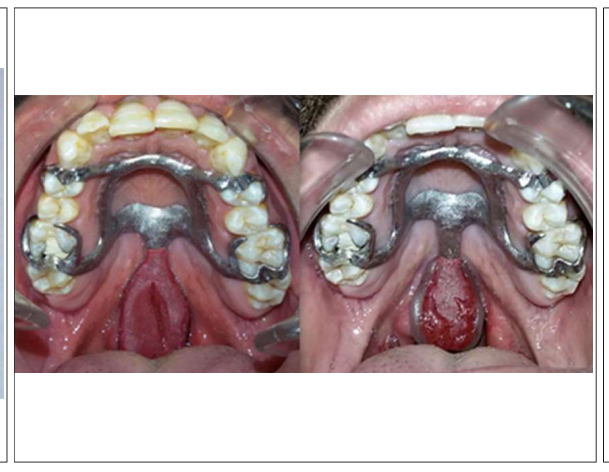

Figure 2: Making of impression of pharyngeal defect.

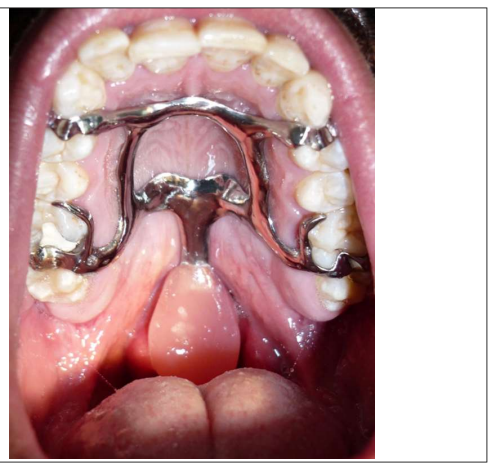

Figure 3: Final pharyngeal obturator. 
speech therapy is the treatment of choice. Yamaguchi et al. mentioned various surgical options, e.g. pharyngeal flap, sphincter pharyngoplasty, posterior pharyngeal wall augmentation and Furlow double opposing Z-plasty. ${ }^{8}$

The pharyngeal obturator only replaces the missing part of the soft palate, so it should not displace the soft palate musculature. To attain this purpose, selection of type of final impression material is of utmost importance. In this case, low fusing impression compound was used alongwith tissue conditioner for final border molding. Other materials available for final border molding are impression waxes, elastomeric impression materials such as polyvinylsiloxane, polyether and zinc oxide eugenol. Zinc oxide eugenol is excellent impression material with only $0.1 \%$ dimensional changes during setting, but it becomes brittle after setting, so needs to be handled carefully. If a broken piece of zinc oxide eugenol is aspirated, it can lead to life-threatening complications. ${ }^{3,6}$ Kahlon et al. reported that permanent soft silicone lining material can be used for speech bulb fabrication which can be more patient friendly. ${ }^{9}$

Pharyngeal prosthesis consists of two parts. One is oral or palatal and the other is pharyngeal or obturator part. If maxillary dentition is intact, retention of the prosthesis is provided by direct and indirect retainers as in cast metal RPD frameworks. In case of missing maxillary dentition, retention for the prosthesis becomes a challenge. In such cases, residual ridge and endosseous implants are used for retention of the prosthesis. Occlusal rests, major connector and denture bases are responsible for support of the prosthesis. Stability is provided by major and minor connectors along with denture flanges. Occlusion must be given unmitigated attention. 3,5

Maxillofacial prosthetic treatment of patients with acquired or congenital defects should not only provide physical and functional improvements but also psychological support. Worldwide, only $20 \%$ of cleft palate teams consider the psychological assessment of these patients. Although prosthetic rehabilitation causes patients to feel more normal with better speech and facial features, but there is a need to provide psychological intervention to these patients on regular basis. ${ }^{10}$

In this case, patient with congenital velopharyngeal insufficiency was treated successfully by pharyngeal obturator prosthesis. Emphasis is given to provision of psychological rehabilitation to patients with craniofacial deformities alongwith physical and functional rehabili- tation. Prosthetic management of the velopharyngeal defect is an acceptable treatment option, if surgical options are contra-indicated or refused by the patient.

\section{PATIENTS' CONSENT:}

Written informed consent was taken from patient.

\section{CONFLICT OF INTEREST:}

Authors declared no conflict of interest.

\section{AUTHORS' CONTRIBUTION:}

MWUK: Conception, treatment planning and execution, manuscript writting.

MAM: Treatment planning and execution, literature review, manuscript writting

AAS: Proofreading and review.

\section{REFERENCES}

1. Kazmi SM, Iqbal Z. Prosthodontic management of palatopharyngeal incompetency - A case report on palatal lift prosthesis. J Coll Physicians Surg Pak 2017; 27:S131-3.

2. Raol N, Hartnick CJ. Anatomy and physiology of velopharyngeal closure and insufficiency. Surgery for pediatric velopharyngeal insufficiency. Karger publishers 2015; 76:1-6.

3. Tuna SH, Pekkan G, Gumus HO, Aktas A. Prosthetic rehabilitation of velopharyngeal insufficiency: Pharyngeal obturator prostheses with different retention mechanisms. Eur J Dent 2010; 4:81-7.

4. Ayliff BW, Bressmann T, Al Mardini M, Jokstad A. Evaluation of a modular palatal lift prosthesis with a silicone velar lamina for hypernasal patients. J Prosthet Dent 2014; 112:663-71.

5. Shetty NB, Shetty S, Nagraj E, D'Souza R, Shetty O. Management of velopharyngeal defects: A review. J Clin Diagn Res 2014; 8:283-7.

6. Keyf F, Sahin N, Aslan Y. Alternative impression technique for a speech-aid prosthesis. Cleft Palate Craniofac J 2003; 40: 566-8.

7. Bou C, Liang AF, Plaire V, Naveau A. A new membrane obturator prosthesis concept for soft palate defects. Int $J$ Prosthodont 2018; 31:584-6.

8. Yamaguchi $\mathrm{K}$, Lonic $\mathrm{D}$, Lee $\mathrm{CH}$, Wang $\mathrm{SH}$, Yun C, Lo LJ. A treatment protocol for velopharyngeal insufficiency and the outcome. Plast Reconstr Surg 2016; 138:290e-9e.

9. Kahlon SS, Kahlon M, Gupta S, Dhingra PS. The soft palate friendly speech bulb for velopharyngeal insufficiency. J Clin Diagn Res 2016; 10:ZD01-02.

10. Hickey AJ, Salter M. Prosthodontic and psychological factors in treating patients with congenital and craniofacial defects. J Prosthet Dent 2006; 95:392-6. 